\title{
Heart Rate Asymmetry in Response to Colored Light
}

\author{
Saman Parvaneh ${ }^{1,2}$, Nader Jafarnia Dabanloo ${ }^{1}$, Shahab Rezaei $^{3}$, Sadaf Moharreri $^{4}$, \\ Nima Toosizadeh ${ }^{5}$ \\ ${ }^{1}$ Islamic Azad University, Science and Research Branch, Tehran, Iran \\ ${ }^{2}$ Philips Research North America, Cambridge, MA, USA \\ ${ }^{3}$ Islamic Azad University, Central Tehran Branch, Tehran, Iran \\ ${ }^{4}$ Islamic Azad University, Khomeini Shahr Branch, Isfahan, Iran \\ ${ }^{5}$ University of Arizona, Department of Medicine and Biomedical Engineering, Tucson, AZ, USA
}

\begin{abstract}
Heart rate asymmetry (HRA) quantifies imbalance of heart rate acceleration and deceleration. The impact of different colored lights on autonomic nervous system captured by HRA was studied in this paper. Using electrocardiogram (ECG) data for 16 female volunteers exposed to four different colored lights (red, green, blue, and yellow), three common HRA indices (Porta Index-PI, Guzik Index-GI, and Slope Index-SI) were calculated. Analysis of data revealed a statistically significant difference in PI (p<0.001), GI $(p=0.006)$, and SI $(p=0.001)$ depending on which colored light was used as visual stimuli. These results propose that HRA can be used as a complementary measure along with heart rate and heart rate variability to quantify heart response to colored light stimulation.
\end{abstract}

\section{Introduction}

Every visual stimulus process by the human perceptual system contains color information and blue, yellow, red, and green are considered as the basic colors in the psychology [1, 2].

It has been shown that different colored light can cause changes in physiological responses such as heart rate (HR) and heart rate variability (HRV) [1-7]. On other hand, the Autonomic Nerve System (ANS) is responsible for shortterm regulation of the $\mathrm{HR}, \mathrm{HRV}$, and blood pressure. Therapies using colored light (Chromotherapy) could potentially be used for ANS control.

Heart rate asymmetry (HRA) is a new approach to quantify changes (e.g., increase or decrease) in heart rate with respect to previous heart beat [8-10] and is shown to be different in diabetes patients compared to healthy individuals [11] as well as in people with different stress level [12].

Changes in HR and HRV in response to colored light has been studied previously [1-7], but little is known about how HRA will change in response to colored visual stimuli. Therefore, the purpose of this study was to evaluate the impact of different colored light on ANS captured by HRA to study the differences between acceleration and deceleration in heart rate in response to different colors.

\section{Data and Method}

\subsection{Data}

ECG data (lead II with sampling frequency equal to 200 $\mathrm{Hz}$ ) were recorded from 16 female volunteers (Age: $24.75 \pm 1.43$ ), while they were exposed to four different colored lights, including red, green, blue, and yellow.

For color stimuli, computer screen was placed one meter away from the subject and other confounding factors such as noise, environmental light, and smell were controlled. Each color was presented for five minutes within a randomized order. Between each color stimuli, a resting time of 10 minutes was given to cancel the effects of previous stimulation. Of note, all recording was done while participants were seated on a chair.

\subsection{Method}

\subsubsection{QRS Detection}

The Pan-Tompkins algorithm was used for QRS detection [13]. As suggested in the guideline [14], the output of the QRS detection algorithm manually checked by an expert. RR interval series were created using corrected QRS peaks.

\subsubsection{Heart Rate Asymmetry}

HRA is defined as uneven distribution of points above 
and below identity line (IL) $[9,10]$ and can be visually observed in Poincaré plot, plot of each RR interval against next RR interval.

If we represent $n^{\text {th }} R R$ interval as $R R_{n}$ and define $\Delta R$ as $\mathrm{RR}_{\mathrm{n}+1}-\mathrm{RR}_{\mathrm{n}}$, IL in Poincaré Plot corresponds to $\Delta \mathrm{R}=0$. Also, deceleration and acceleration of heart rate correspond to points above IL $(\mathrm{PA}=\Delta \mathrm{R}>0)$ and below $\mathrm{IL}(\mathrm{PB}=\Delta \mathrm{R}<0)$, respectively. In this paper, Porta Index [15], Guzik Index [10], and Slope Index [9] were calculated within five minutes time windows across stimulations with different colored light to quantitatively represent HRA:

1- Porta Index (PI): an index that represents prevalence of acceleration of heart rate based on quantifying the distribution of points in Poincaré Plot with respect to IL [15]:

$$
P I,[\%]=\frac{n_{P B}}{n_{P A}+n_{P B}} \times 100
$$

where, $n_{P A}$ and $n_{P B}$ are number of points above and below IL.

2- Guzik Index (GI): is defined as a percentage of the distance of points above IL with respect to the total distance of all points with respect to IL [10]:

$$
G I,[\%]=\frac{\sum_{i=1}^{n_{P A}} d_{i}^{+}}{\sum_{i=1}^{n_{P A}} d_{i}^{+}+\sum_{i=1}^{n_{P B}} d_{i}^{-}} \times 100
$$

where, $d_{i}^{+}$and $d_{i}^{-}$are distances of points above and below IL from identity line, respectively.

3- Slope Index (SI): SI is defined as a percentage of phase angle difference of points above IL with respect to the total phase angle difference [9]:

$$
S I,[\%]=\frac{\sum_{i=1}^{n_{P A}} \Delta \theta_{i}}{\sum_{i=1}^{n_{P A}} \Delta \theta_{i}+\sum_{i=1}^{n_{P B}} \Delta \theta_{i}} \times 100
$$

where, $\quad \Delta \theta_{i}=\theta_{I L}-\theta_{i}=45^{\circ}-\theta_{i} \quad$ and $\theta_{i}=\operatorname{atan}\left(\frac{R R_{n+1}}{R R_{n}}\right) . \quad \theta_{i}$ is the phase angle of consecutive RR intervals $\left(R R_{n}, R R_{n+1}\right)$.

\subsubsection{Statistical Analysis}

Mean and standard deviation were reported for all HRA features. HRA for exposure to different colored light were compared using Friedman test followed by Post hoc analysis with Wilcoxon signed-rank tests with a Bonferroni correction applied, resulting in a statistically significant level set at $p$-value $<0.008$. SPSS was used for all statistical analysis (IBM, version 24, Chicago, IL).

\section{Results and Discussion}

Mean and standard deviation of HRA indexes in response to different colored lights are reported in Table 1.
Furthermore, the changes in these parameters across groups with different color stimuli are visually depicted in Figure 1.

Friedman test revealed a statistically significant difference in PI $(\mathrm{p}<0.001)$, GI $(\mathrm{p}=0.006)$, and SI $(\mathrm{p}=0.001)$ depending on which colored light was used as visual stimuli. The $p$-values for pairwise comparison of HRA indexes are reported in Table 2. PI was significantly different between red and yellow, green and blue, green and yellow, and blue and yellow. Also, SI was significantly different between red and blue, green and blue, and blue and yellow. Despite an overall increase in GI from red to green and blue, there were no significant differences between red and green, red and blue, and green and blue.

Table 1. Mean and standard deviation of HRA indexes across groups with different colored light stimuli (PI: Porta Index, GI: Guzik Index, SI: Slope Index)

\begin{tabular}{cccc}
\cline { 2 - 4 } & \multicolumn{3}{c}{ HRA Indexes } \\
\cline { 2 - 4 } Red & PI, [\%] & GI, [\%] & SI, [\%] \\
\hline Green & $49.52 \pm 2.17$ & $45.75 \pm 1.72$ & $44.95 \pm 2.16$ \\
\hline Blue & $45.82 \pm 1.38$ & $46.85 \pm 1.21$ & $45.26 \pm 1.76$ \\
\hline Yellow & $39.49 \pm 2.91$ & $46.26 \pm 1.01$ & $39.54 \pm 3.96$ \\
\hline
\end{tabular}

Table 2. Wilcoxon signed-rank tests for pairwise comparison of the HRA indexes for between group differences. Statistically significant ( $p$-value $<0.008$ after using Bonferroni adjustment) marked in bold (PI: Porta Index, GI: Guzik Index, SI: Slope Index).

\begin{tabular}{cccc}
\cline { 2 - 4 } & \multicolumn{3}{c}{ HRA Indexes } \\
\cline { 2 - 4 } & PI, [\%] & GI, [\%] & SI, [\%] \\
\hline Red \& Green & 0.02 & 0.02 & 0.88 \\
\hline Red \& Blue & 0.04 & 0.02 & $<\mathbf{0 . 0 5}$ \\
\hline Red \& Yellow & $<\mathbf{0 . 0 5}$ & 0.06 & 0.44 \\
\hline Green \& Blue & $<\mathbf{0 . 0 5}$ & 0.06 & $<0.05$ \\
\hline Green \& Yellow & $<\mathbf{0 . 0 5}$ & 0.62 & 0.88 \\
\hline Blue \& Yellow & $<\mathbf{0 . 0 5}$ & 0.01 & $<\mathbf{0 . 0 5}$ \\
\hline
\end{tabular}

The reduction in PI in yellow light stimuli compared to red, blue and green indicates that prevalence of acceleration in yellow light is higher than other light conditions. Furthermore, reduction in GI in red light compared to green and blue lights is an indication of acceleration contribution to magnitude asymmetry. The result of this study also suggest that blue light can cause a phase asymmetry.

Previous research confirmed changes in HRV in response to colored light [3-6]. This study is the first study that confirms the changes in HRA in response to external colored lights. However, since the impact of various physiological mechanisms on HRA as a new measure is not clear, further research is needed to better understand the relationship between these changes in response to external environmental factors (e.g., light). The promising results of this study and previous reports showing changes 
in HR, HRV, and HRA in response to colored light warrant future research on studying ANS through these measures in response to colored light stimulation (Chromotherapy) for ANS control.

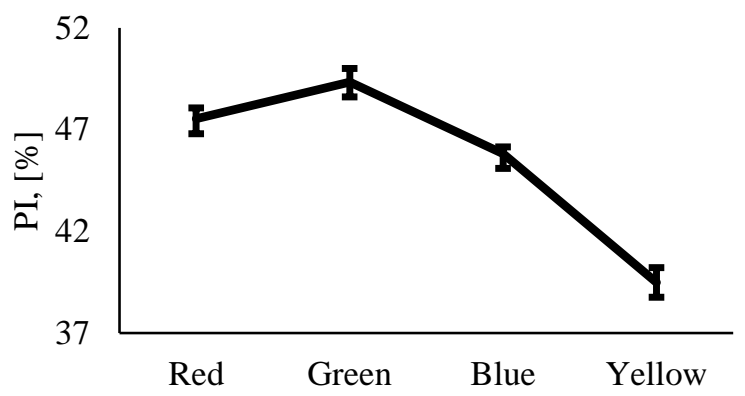

(a)

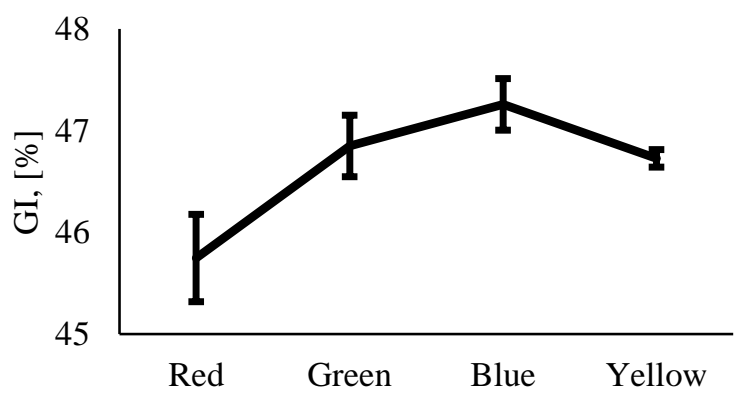

(b)

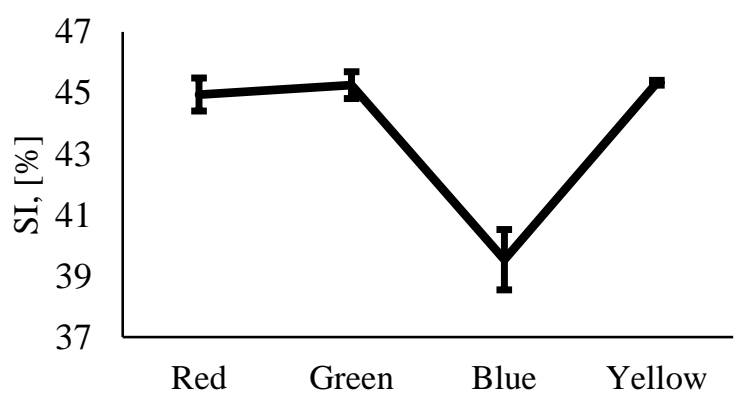

(c)

Figure 1. Porta Index (PI), Guzik Index (GI), and Slope Index (SI) for groups with different colored light stimuli (mean values and standard errors are presented).

\section{Conclusion}

In this article, heart rate asymmetry as an indicator of imbalance between acceleration and deceleration of heart rate was studied across groups with different colored light stimuli. Results of this study suggest that heart rate asymmetry behavior differs in response to different colored lights. Therefore, it can be used in conjunction with heart rate variability parameters for capturing response to Chromotherapy. We speculate that reduction in PI and SI in blue compared to red and green color may be linked to reduction in activity of parasympathetic nervous system.

\section{References}

[1] K. Jacobs and J. Suess, "Effects of four psychological primary colors on anxiety state," Perceptual and motor skills, vol. 41, p. 207, 1975.

[2] S. Moharreri, N. Jafarnia Dabanloo, S. Parvaneh, and A. M. Nasrabadi, "How to Interpret Psychology from Heart Rate Variability?," in Middle East Conference on Biomedical Engineering (MECBME), Sharjah, UAE, 2011.

[3] S. Moharreri, N. J. Dabanloo, S. Parvaneh, and A. M. Nasrabadi, "The Relation between Colors, Emotions and Heart using Triangle Phase Space Mapping (TPSM)," Computers in Cardiology, vol. 38, 2011.

[4] A. Schäfer and K. W. Kratky, "The effect of colored illumination on heart rate variability," Complementary Medicine Research, vol. 13, pp. 167-173, 2006.

[5] S. Moharreri, S. Rezaei, N. J. Dabanloo, and S. Parvaneh, "Study of induced emotion by color stimuli: Power spectrum analysis of heart rate variability," in Computing in Cardiology Conference (CinC), 2014, 2014, pp. 977-980.

[6] S. Moharreri, N. J. Dabanloo, S. Parvaneh, A. M. Nasrabadi, and G. Attarodi, "Personality psychology using heart responses to color stimulus," in Computing in Cardiology, 2011, 2011, pp. 97-100.

[7] C.-J. Choi, K.-S. Kim, C.-M. Kim, S.-H. Kim, and W.-S. Choi, "Reactivity of heart rate variability after exposure to colored lights in healthy adults with symptoms of anxiety and depression," International Journal of Psychophysiology, vol. 79, pp. 83-88, 2011.

[8] P. Guzik, J. Piskorski, T. Krauze, A. Wykretowicz, and H. Wysocki, "Heart rate asymmetry by Poincaré plots of RR intervals," Biomedizinische Technik, vol. 51, pp. 272-275, 2006.

[9] C. Karmakar, A. Khandoker, and M. Palaniswami, "Phase asymmetry of heart rate variability signal," Physiological measurement, vol. 36, p. 303, 2015.

[10] J. Piskorski and P. Guzik, "Geometry of the Poincaré plot of RR intervals and its asymmetry in healthy adults," Physiological measurement, vol. 28, p. 287, 2007.

[11] P. Guzik, J. Piskorski, P. Contreras, and E. R. Migliaro, "Asymmetrical properties of heart rate variability in type 1 diabetes," Clinical Autonomic Research, vol. 20, pp. 255-257, 2010.

[12] S. Parvaneh, N. Toosizadeh, and S. Moharreri, "Impact of mental stress on heart rate asymmetry," in Computing in Cardiology Conference (CinC), 2015, 2015, pp. 793-796.

[13] J. Pan and W. J. Tompkins, "A real-time QRS detection algorithm," Biomedical Engineering, IEEE Transactions on, pp. 230-236, 1985.

[14] M. Malik, "Heart rate variability," Annals of Noninvasive Electrocardiology, vol. 1, pp. 151-181, 1996.

[15] A. Porta, K. R. Casali, A. G. Casali, T. Gnecchi-Ruscone, E. Tobaldini, N. Montano, et al., "Temporal asymmetries of shortterm heart period variability are linked to autonomic regulation," American Journal of Physiology-Regulatory, Integrative and Comparative Physiology, vol. 295, pp. R550-R557, 2008.

Address for correspondence.

Saman Parvaneh

2 Canal Park, 3rd floor, Cambridge, MA 02141

parvaneh@ieee.org 
Page 4 